\title{
High Grade Appendix Mucinous Adenocarcinoma
}

National Cancer Institute

\section{Source}

National Cancer Institute. High Grade Appendix Mucinous Adenocarcinoma. NCI

Thesaurus. Code C96421.

A mucinous adenocarcinoma that arises from the appendix. The malignant cells are numerous and exhibit high grade dysplasia. It may be associated with pseudomyxoma peritonei, but unlike the low grade mucinous neoplasm, it invades other organs and metastasizes to distant anatomic sites. 